\title{
Solution of a generalized Stieltjes problem
}

\author{
B Sriram Shastry ${ }^{1,2}$ and Abhishek Dhar ${ }^{3,4}$ \\ ${ }^{1}$ Bell Laboratories Lucent Technologies, 600 Murray Hill, NJ 07974, USA \\ 2 Indian Institute of Science, Bangalore 560012, India \\ ${ }^{3}$ Raman Research Institute, Bangalore 560080, India \\ ${ }^{4}$ Poornaprajna Institute, Bangalore, India
}

Received 31 January 2001

Published 27 July 2001

Online at stacks.iop.org/JPhysA/34/6197

\begin{abstract}
We present the exact solution for a set of nonlinear algebraic equations $\frac{1}{z_{l}}=\pi d+\frac{2 d}{n} \sum_{m \neq l} \frac{1}{z_{l}-z_{m}}$. These were encountered by us in a recent study of the low-energy spectrum of the Heisenberg ferromagnetic chain. These equations are low- $d$ (density) 'degenerations' of a more complicated transcendental equation of Bethe's ansatz for a ferromagnet, but are interesting in themselves. They generalize, through a single parameter, the equations of Stieltjes, $x_{l}=\sum_{m \neq l} 1 /\left(x_{l}-x_{m}\right)$, familiar from random matrix theory. It is shown that the solutions of these set of equations are given by the zeros of generalized associated Laguerre polynomials. These zeros are interesting, since they provide one of the few known cases where the location is along a nontrivial curve in the complex plane that is determined in this work. Using a 'Green function' and a saddle point technique we determine the asymptotic distribution of zeros.
\end{abstract}

PACS numbers: 02.10.-v, 05.50.+q, 75.10.Hk, 75.30.-m

\section{Introduction}

The study of nonlinear algebraic sets of equations that arise in various physical contexts is a rich field of study. A famous example is the problem of Stieltjes, namely the set of equations $x_{l}=\sum_{m \neq l} 1 /\left(x_{l}-x_{m}\right)$, for $n$ variables $x_{l}$, that arises when we consider the stationary points of the random matrix Gaussian ensemble 'action' $1 / 2 \sum x_{l}^{2}-1 / 2 \sum_{l \neq m} \log \left(\left|x_{l}-x_{m}\right|\right)$ [2]. In that context, the well known result is that the $x_{l}$ are all real, and further, are the roots of Hermite's polynomials of degree $n$, forming a dense set along the real line with the familiar semicircular density of states as $n \rightarrow \infty$.

In a recent study of the 'most elementary excitations' of the Heisenberg ferromagnet in one dimension [1], we came across a one-parameter generalization of the Stieljes problem

$$
\frac{1}{z_{l}}=\pi d+\frac{2 d}{n} \sum_{m \neq l} \frac{1}{z_{l}-z_{m}} .
$$


where the single parameter $d(0 \leqslant d \leqslant 1)$ has the physical significance of the density of (hardcore) particles on a lattice. By a simple redefinition of variables (see below), this is recognizable as a generalized Stieltjes problem $\frac{\beta_{l}}{1+i \lambda \beta_{l}}=\frac{1}{n} \sum_{m \neq l} \frac{1}{\beta_{l}-\beta_{m}}$, where, as the parameter $\lambda$ vanishes, it reduces to the standard problem.

We found the equation quite fascinating in its own terms, requiring some new tricks to solve. It further appears that this problem connects with [4, 8-11] that of the asymptotic distribution of zeros of the standard polynomials (orthogonal in most instances though not in the present case). It provides an explicit example of a case where the zeros live on a nontrivial curve in the complex plane, which is determined here.

The paper is organized as follows. In section 2 we obtain a perturbative solution of equation (1) while in section 3 we give the exact solution. The construction of the 'Green function' and the determination of the distribution of zeros are described in section 4 . We summarize our results in section 5. Some results of Stieltjes on the properties of zeros of orthogonal polynomials are described in appendix A. In appendix B we describe a second method of obtaining the Green function.

\section{The perturbative solution}

We first present a 'perturbative solution' in a parameter displayed below, perturbing around the Stieltjes example. In equation (1) we make a change of variables $z_{l}=\left(1+\mathrm{i} \lambda \beta_{l}\right) /(\pi d)$, with $\lambda=\sqrt{2 d}$. The new variable $\beta_{l}$ satisfies the following equation:

$$
\frac{\beta_{l}}{1+\mathrm{i} \lambda \beta_{l}}=\frac{1}{n} \sum_{m \neq l} \frac{1}{\beta_{l}-\beta_{m}} \text {. }
$$

We note that for $\lambda=0$, this equation reduces to the well known form whose solution can be found in terms of the roots of the Hermite polynomials, the example of Stieltjes [3]. The general formalism for this is summarized and expanded upon in appendix A. Thus one obtains $\beta_{l}=x_{l}$ where $x_{l}$ satisfy $H_{n}\left(\sqrt{n} x_{l}\right)=0$, where $H_{n}$ is the Hermite polynomial of degree $n$. The division by $n$ in equation (2) is our preference, since it enables an easier passage to the 'thermodynamic limit' $n \rightarrow \infty$, and we discuss this feature later.

One possible approach is to try for a perturbative solution around the known result of Stieltjes to various orders in the small parameter $\lambda$ (see below). The perturbation analysis requires a knowledge of the eigenvectors and eigenvalues of an interesting matrix studied by Calogero (we call this the Calogero matrix) in which the matrix elements are algebraic functions of $x_{l}$, the roots of the Hermite polynomials.

For $\lambda \neq 0$ we try a perturbation series solution of the form $\beta_{l}=\sum_{k} \beta_{l}^{(k)}(\mathrm{i} \lambda)^{k}$. Putting this in equation (1) and matching terms order by order we obtain the following set of equations for the unknown coefficients $\beta_{l}^{(k)}$ :

$$
\begin{gathered}
\beta_{l}^{(0)}-\frac{1}{n} \sum_{m \neq l} \frac{1}{\beta_{l}^{(0)}-\beta_{m}^{(0)}}=0 \quad \beta_{l}^{(1)}+\frac{1}{n} \sum_{m \neq l} \frac{\beta_{l}^{(1)}-\beta_{m}^{(1)}}{\left[\beta_{l}^{(0)}-\beta_{m}^{(0)}\right]^{2}}=\left[\beta_{l}^{(0)}\right]^{2} \\
\beta_{l}^{(2)}+\frac{1}{n} \sum_{m \neq l} \frac{\beta_{l}^{(2)}-\beta_{m}^{(2)}}{\left[\beta_{l}^{(0)}-\beta_{m}^{(0)}\right]^{2}}=2 \beta_{l}^{(1)} \beta_{l}^{(0)}-\left[\beta_{l}^{(0)}\right]^{3}+\frac{1}{n} \sum_{m \neq l} \frac{\left[\beta_{l}^{(1)}-\beta_{m}^{(1)}\right]^{2}}{\left[\beta_{l}^{(0)}-\beta_{m}^{(0)}\right]^{3}} \\
\beta_{l}^{(3)}+\frac{1}{n} \sum_{m \neq l} \frac{\beta_{l}^{(3)}-\beta_{m}^{(3)}}{\left[\beta_{l}^{(0)}-\beta_{m}^{(0)}\right]^{2}}=2 \beta_{l}^{(2)} \beta_{l}^{(0)}+\left[\beta_{l}^{(1)}\right]^{2}-3 \beta_{l}^{(1)}\left[\beta_{l}^{(0)}\right]^{2}+\left[\beta_{l}^{(0)}\right]^{4} \\
+\frac{2}{n} \sum_{m \neq l} \frac{\left[\beta_{l}^{(2)}-\beta_{m}^{(2)}\right]\left[\beta_{l}^{(1)}-\beta_{m}^{(1)}\right]}{\left[\beta_{l}^{(0)}-\beta_{m}^{(0)}\right]^{3}}-\frac{1}{n} \sum_{m \neq l} \frac{\left[\beta_{l}^{(1)}-\beta_{m}^{(1)}\right]^{3}}{\left[\beta_{l}^{(0)}-\beta_{m}^{(0)}\right]^{4}} .
\end{gathered}
$$


As noted above, the lowest-order solution is given by $\beta_{l}^{(0)}=x_{l}$ where $H_{n}\left(\sqrt{n} x_{l}\right)=0$. It turns out that it is possible to solve the equations of the perturbative series at every order. To see this we first define the Calogero matrix [5]:

$$
T_{l m}=\delta_{l m} \sum_{j \neq l} \frac{1}{\left(x_{l}-x_{j}\right)^{2}}-\left(1-\delta_{l m}\right) \frac{1}{\left(x_{l}-x_{m}\right)^{2}} .
$$

We then note that the set of equations (3), for $k>0$, have the following general structure:

$$
\beta_{l}^{(k)}+\frac{1}{n} \sum_{m} T_{l m} \beta_{m}^{(k)}=g_{l}^{(k)}
$$

where the function $g_{l}^{(k)}$ appearing at $k$ th order in perturbation is a function of lower-order terms only and so is known. Hence at every stage we have to essentially solve a linear matrix equation with the same matrix appearing at all orders. This can be done by using some rather special properties of the Calogero matrix, $T$. The following result is easily obtained:

$T_{l m} x_{m}^{r}=r x_{l}^{r}-\frac{(r-1)(2 n-r)}{2} x_{l}^{r-2}-\sum_{s=1}^{[r / 2-1]}(r-2 s-1)\left(\sum_{m=1}^{n} x_{m}^{2 s}\right) x_{l}^{r-2 s-2}$

where $[p]$ denotes the largest integer $\leqslant p$. Note that the rhs of equation (6) involves powers of $x_{l}$ alone. It is also possible to show that at every order the function $g_{l}^{(k)}$ is a known polynomial of degree $(k+1)$ in the variable $x_{l}$. Thus it follows that the $k$ th-order solution can be obtained in the form

$$
\beta_{l}^{(k)}=\sum_{r=0}^{k+1} c_{r}^{(k)} x_{l}^{r}
$$

where the coefficients $c_{r}^{(k)}$ are obtained by inserting the above form into equation (5), using the property equation (6) of the Calogero matrix $T$ and then comparing the lhs and rhs of the equation. To second order we get

$\beta_{l}=x_{l}+\mathrm{i} \lambda\left[\frac{1}{3} x_{l}^{2}+\frac{1}{3}\left(1-\frac{1}{n}\right)\right]+(\mathrm{i} \lambda)^{2}\left[\frac{1}{36} x_{l}^{3}+\frac{1}{72}\left(14-\frac{11}{n}\right) x_{l}\right]+\mathrm{O}\left(\lambda^{3}\right)$.

It is straightforward, though tedious, to carry the perturbation to any order. We have been unable to find a closed form for the general term.

As an example of this perturbation theory we note that the first-order term in $\lambda$ is already enough to compute several objects of interest. In the ferromagnet problem, one needs the 'energy' of the state $w$, defined by $w=4 /(n d) \sum_{l} 1 / z_{l}^{2}$ (the present $w=n \epsilon / d J$ of [1]). To obtain this energy to order $d^{2}$ the perturbation series to first order in $\lambda$ suffices. In the limit of large $n$ the $x_{l}$ form a continuum stretching from $-\sqrt{2}$ to $\sqrt{2}$ with the familiar semicircular density of states $\rho(x)=\frac{1}{\pi} \sqrt{2-x^{2}}$. This solution can be used to obtain the energy to order $d^{2}$. The energy $w$ for low $d$ can be found as $w=4 \pi^{2}\left[d-d^{2}\left\{6\left\langle\left(\beta_{l}^{(0)}\right)^{2}\right\rangle+4\left\langle\beta_{l}^{(1)}\right\rangle\right\}+\mathrm{O}\left(d^{3}\right)\right]$, where the averages are normalized sums over the indicated variables. Using the explicit expression equation (8) and converting the sums to integrals over the semicircular density of states we finally get the low-density formula

$$
w=4 \pi^{2} d(1-d)+\mathrm{O}\left(d^{3}\right) .
$$

\section{Exact solution}

The exact solution of equation (1) can be obtained using the results of Stieltjes, which are described in appendix A. We will give two different but related solutions. The first 
one is directly related to the perturbative approach. We first note that after the change of variables $u_{l}=\sqrt{n} \beta_{l}$, equation (2) becomes of the form equation (A.8) with $p(u)=$ $\left(1+\mathrm{i}(2 d / n)^{1 / 2} u\right) ; q(u)=-2 u$. The $\left\{u_{l}\right\}$ are then obtained as zeros of the the corresponding polynomial function $g(u)$, which in this case satisfies the following differential equation:

$$
(1+\mathrm{i} v u) g^{\prime \prime}(u)-2 u g(u)+2 n g(u)=0
$$

where $v=\sqrt{2 d / n}$. For $v=0$ this is just Hermite's equation and we recover our zeroth-order perturbative result. For $v \neq 0$, we obtain the following series solutions (which we denote by $\left.Q_{n}(u)\right)$ :

$$
Q_{n}(u)=\sum_{k=0}^{n} c_{k} u^{k}
$$

with

$$
\begin{aligned}
& c_{k}=\frac{-(k+2)(k+1)}{2(n-k)} c_{k+2}-\frac{\mathrm{i} \nu k(k+1)}{2(n-k)} c_{k+1} \\
& c_{n-1}=\frac{-\mathrm{i} \nu n(n-1) c_{n}}{2} \quad c_{n}=2^{n} .
\end{aligned}
$$

To obtain our second solution to equation (1), we make the change of variables $z_{l}=$ $-y_{l} /(\pi n)$, so that equation (1) is transformed to

$$
\frac{n / d+y_{l}}{y_{l}}=2 \sum_{m \neq l} \frac{1}{y_{l}-y_{m}} .
$$

This is in a form where we can once again apply Stieltjes' result. The corresponding differential equation in this case is the associated Laguerre equation (A.11). Thus $\left\{z_{l}\right\}$ are obtained as zeros of the associated Laguerre polynomials $L_{n}^{(-n / d-1)}(-n \pi z)[6]^{5}$. Note that the usual orthogonal Laguerre polynomials $L_{n}^{a}(y)$ have $a>-1$, while in our case $a=-(n / d+1)<-(n+1)$. In this case it can be proved [4] that for $n$ even there are no real zeros while for odd $n$ there is a single real zero. Physically this corresponds to the fact that the single-particle potential (see appendix A) in this case is no longer confining and therefore we cannot get any position of equilibrium for the particles.

The two solutions described are related as

$$
Q_{n}(x)=\frac{(-\mathrm{i})^{\mathrm{n}} \mathrm{n} ! 2^{\mathrm{n} / 2}}{(n / d)^{n / 2}} L_{n}^{-n / d-1}\left(-\mathrm{i}(2 n / d)^{1 / 2} x-n / d\right) .
$$

We note that since in the limit $d \rightarrow 0$ we get $Q_{n}(x) \rightarrow H_{n}(x)$, this leads to the following interesting identity relating the Hermite and Laguerre polynomials:

$$
\lim _{d \rightarrow 0} \frac{(-\mathrm{i})^{\mathrm{n}} \mathrm{n} ! 2^{\mathrm{n} / 2}}{(n / d)^{n / 2}} L_{n}^{-n / d-1}\left(-\mathrm{i}(2 n / d)^{1 / 2} x-n / d\right)=H_{n}(x) .
$$

Such a relation is of course well known (see e.g. [7]) for the case of large positive order $m$ in $L_{n}^{m}$.

5 In this paper Muttalib has shown a similar connection between the zeros of Laguerre polynomials and the roots of a set of equations arising in the context of random matrix theory. However he considers real zeros of the Laguerre polynomials while we consider complex zeros of the associated Laguerre polynomials and, as we show, this case turns out to be much more rich and interesting. Also, unlike his case, our equations can be continuously deformed to the classic Stieltjes problem with Hermite polynomials. 


\section{Asymptotic distribution of zeros}

In the previous section it was shown that the solutions to equation (1) are the zeros of generalized associated Laguerre polynomials. Unlike the case of usual orthogonal polynomials where the zeros are always real, in the present case the zeros are complex. Szego [4] has already noted that the zeros of $L_{n}^{-|m|}(z)$ are in general non-real except for at most one root. We found numerically that all the complex roots live on a smooth curve in the complex plane; we now proceed to obtain the distribution of the roots in the complex plane in the asymptotic limit $n \rightarrow \infty$.

Let us define the following Green function (also known as the Stieltjes transform in the mathematics literature):

$$
G(z)=\frac{1}{n} \sum_{l=1}^{n} \frac{1}{z-z_{l}}
$$

An important aspect of our analysis is in the way in which we scale the various arguments by $n$, and normalize by $n$ as above. The remaining variables, such as $G, z$ and $y$ below are of $\mathrm{O}(1)$ as $n \rightarrow \infty$, whereby various simplifications arise for large $n$.

Using the results described in appendix A we get

$$
G(z)=\frac{1}{n F(z)} \frac{\mathrm{d} F(z)}{\mathrm{d} z}=\frac{1}{n} \frac{\mathrm{d}}{\mathrm{d} z} \ln (F(z))
$$

where, in the present case, we have $F(z)=L_{n}^{-n / d-1}(-n \pi z)$. The usual integral equation representation of the associated Laguerre polynomial gives

$$
F(z)=\frac{1}{2 \pi \mathrm{i}} \oint \frac{\exp \left(\frac{n \pi z y}{1-y}\right)}{(1-y)^{-n / d} y^{n+1}} \mathrm{~d} y=\frac{1}{2 \pi \mathrm{i}} \oint \frac{\mathrm{e}^{-n \phi(y, z)}}{y} \mathrm{~d} y
$$

where

$$
\phi(y, z)=\ln (y)-\frac{1}{\mathrm{~d}} \ln (1-y)-\frac{\pi z y}{1-y} .
$$

The contour can be taken as any closed loop around the origin which does not cross the branch line, which we take as the real line from $y=1$ to $\infty$. In the large- $n$ limit the integral can be evaluated by a saddle point method, as is standard in statistical physics. The saddle point of $\phi$ is determined for each $z$ We can choose the contour to pass through the appropriate saddle point. In the usual case, this deformation leads to the asymptotically exact result for the 'free energy'; here we show that it leads to the exact Green function. The saddle-points are determined through $\partial \phi(y, z) / \partial y=0$, which gives

$$
y_{ \pm}=\frac{1-2 d-\pi \mathrm{d} z \pm \pi \mathrm{d} \sqrt{\left(z-z_{+}\right)\left(z-z_{-}\right)}}{2(1-d)}
$$

where

$$
z_{ \pm}=\frac{1-2 d}{\pi d} \pm \frac{\mathrm{i} 2}{\pi} \sqrt{\frac{1}{d}-1}
$$

Of the two saddlepoints we choose the one which gives a smaller value for $\operatorname{Re}[\phi(y, z)]$ since that gives the dominant contribution to the integral. Further it needs to be ensured that it is possible to actually draw a contour, enclosing the origin and not crossing the branch-cut, such that, along the contour, $\phi(y, z)$ takes its minimum value at the saddlepoint. We find that either of the branches can be chosen depending on the location of $z$ in the complex plane. The condition

$$
\operatorname{Re}[\delta \phi]=\operatorname{Re}\left[\phi\left(y_{-}, z\right)-\phi\left(y_{+}, z\right)\right]=0
$$




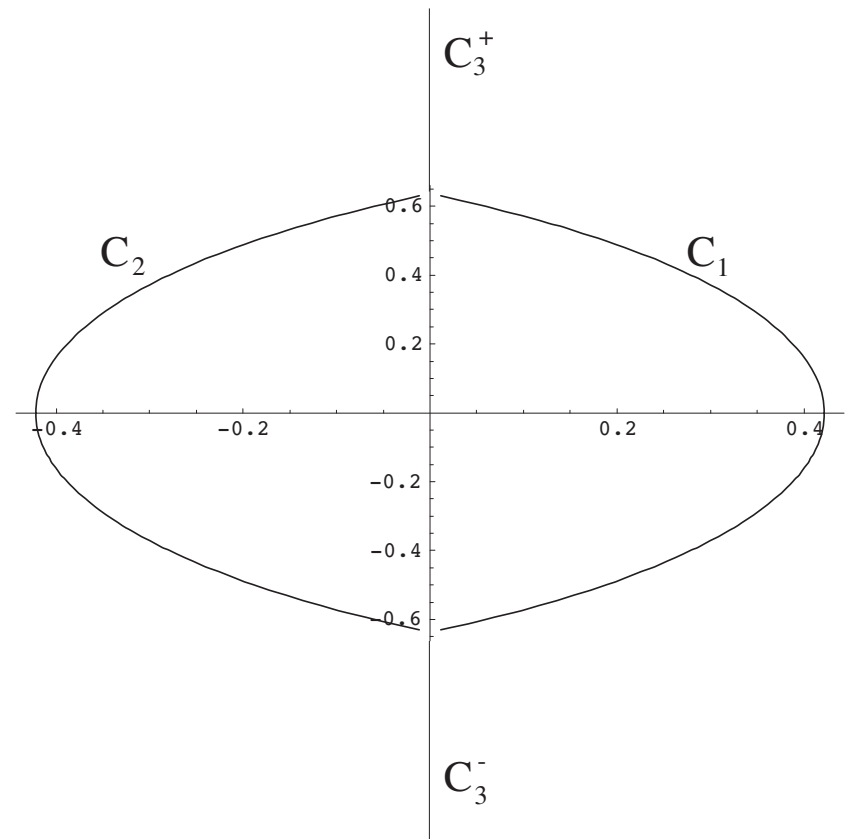

Figure 1. The curves $C_{1}$ and $C_{2}$, in the complex $z$ plane, for density $d=1 / 2$. At this density the curves are explictly given by the formula in equation (22). The curve $C_{1}$ corresponds to the true line of singularities on which the roots lie. There is also a change of branch from $y^{+}$to $y^{-}$as we cross the vertical lines $C_{3}^{ \pm}$from right to left. But there is no discontinuity of the Green function across these lines and hence there are no zeros on these lines.

determines the curve in the $\angle z$ plane on which the roots lie. This condition actually determines two curves $C_{1}$ and $C_{2}$, where $C_{1}$ concaves towards the negative $\operatorname{Re}[z]$ axis (like $x=-y^{2}$ ) and $C_{2}$ concaves in the other direction. The curves $C_{1}$ and $C_{2}$ together form a closed region $R$ in $\angle z$ plane surrounding the point $(1-2 d) /(\pi d)$ (see figure 1 ). To the right of the curve $C_{1}$, the saddlepoint $y_{+}$dominates. As we cross into the region $R$, the saddlepoint $y_{-}$takes over. However when we again cross the curve $C_{2}$, the saddlepoint $y_{-}$continues to determine the free energy even though $\phi\left(y_{+}, z\right)$ is smaller. This is because it is no longer possible to construct the necessary contour through $y_{+}$(this is illustrated in figure 2) and we remain stuck to $y_{-}$. Thus it is the curve $C_{1}$ which determines the locus of roots of the polynomials.

A brief discussion of the curves is in order. In terms of $\kappa=\sqrt{\frac{d}{1-d}}$ we replace $z$ by a variable $\psi$ defined through $z=\frac{1}{\pi \kappa^{2}}\left[\left(1-\kappa^{2}\right)+2 \kappa \sinh (\psi)\right]$ and hence the branch line of the square roots is a curve joining the two points $\psi= \pm \mathrm{i} \pi / 2$. The difference in 'free energies' can be written as

$$
\delta \phi=\mathrm{i} \pi+2 \psi+\frac{2}{\kappa} \cosh (\psi)-\frac{1+\kappa^{2}}{\kappa^{2}} \log \left\{\frac{1+\kappa \exp (\psi)}{1-\kappa \exp (-\psi)}\right\} .
$$

A particularly nice case is that of $d=1 / 2$, where we have $\kappa=1$ and hence another transformation to $b=\left(b_{\mathrm{re}}+\mathrm{i} b_{\text {im }}\right)$ defined through $\sinh (b) \sinh (\psi)=-1$ gives

$$
\delta \phi=\mathrm{i} \pi+2[b-\operatorname{coth}(b)] .
$$

The roots thus live on the curve $b_{\text {re }}=\operatorname{Re} \operatorname{coth}\left(b_{\mathrm{re}}+\mathrm{i} b_{\text {im }}\right)$, which simplifies to the curve

$$
b_{\text {im }}=\frac{1}{2} \arccos \left[\cosh \left(2 b_{\text {re }}\right)-\sinh \left(2 b_{\text {re }}\right) / b_{\text {re }}\right] .
$$




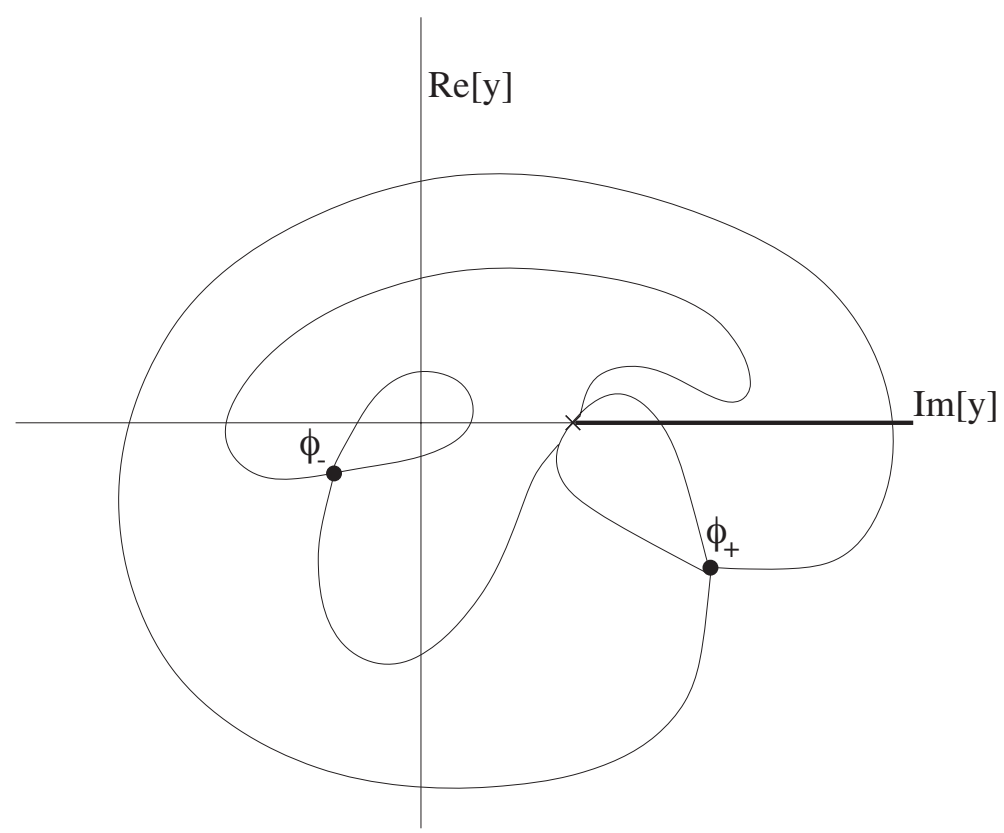

Figure 2. The figure shows, schematically, contours of constant $\phi_{+}$and $\phi_{-}$in the complex $y$ plane (with $d=0.6$ and $z=-0.4+0.3 \mathrm{i}$ ). For this case $\phi_{+}<\phi_{-}$. It is clear that one cannot draw the appropriate contour through the point $y_{+}$and so we are forced to choose the point $y_{-}$even though it has a higher value of $\phi$. This illustrates why there is no change in branch when we cross the curve $C_{2}$.

Note that $z=-2 /(\pi \sinh (b))$, and $b_{\text {re }}$ varies between $b l=0.00079$ and $b u=1.19968$ the values at which the argument of the arccos hits \pm 1 . By choosing the appropriate branch of the arccos function we obtain a smooth curve as depicted in figure 1.

In figure 3 we show curves at different densities, obtained by solving equation (19) numerically. We also plot the roots as obtained from an exact numerical solution of equation (1) for $n=54$.

The asymptotic Green function follows from equation (16):

$$
G(z)=-\left.\frac{\mathrm{d} \phi(y, z)}{\mathrm{d} z}\right|_{y^{ \pm}}=\frac{\pi y_{ \pm}}{1-y_{ \pm}}
$$

where either of the branches $y_{ \pm}$is chosen depending on the value of $z$. A second method of deriving the Green function is described in appendix B.

Density of zeros. The Green function contains complete information on the roots and we now proceed to extract the density of zeros from it. We note that for the case when the roots are all located on the real axis, the density of zeros is given by the discontinuity in the imaginary part of the Green function across the branch line formed by the roots. Here we develop a generalization of this procedure for the case when the roots are distributed on a curve.

We first assume that the curve can be parametrized by a continuous variable $s$ so that points on it are given by $z(s)=(x(s), y(s))$. The Green function is given by

$$
G(z)=\frac{1}{n} \sum_{l} \frac{1}{z-z\left(s_{l}\right)} .
$$




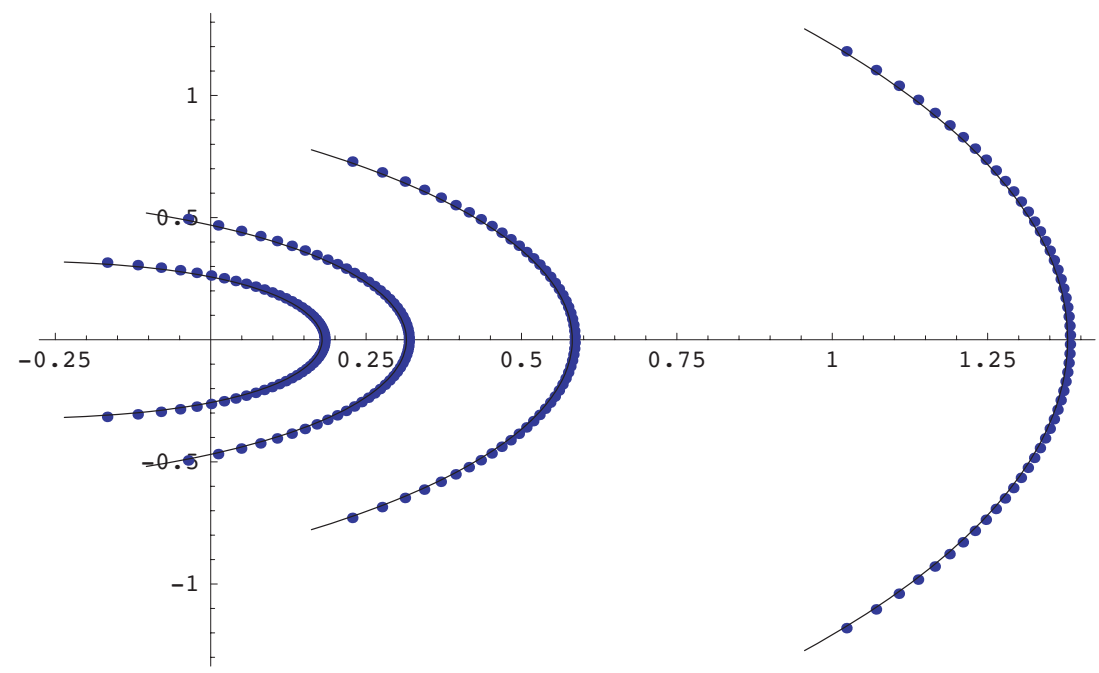

Figure 3. The solid curves give the asymptotic distribution of zeros in the complex $z$-plane obtained from a numerical solution of equation (19) for $d=0.2,0.4,0.6$ and 0.8 (extreme left). Also shown are numerically obtained roots (points) for $n=54$.

We now define the following limiting function:

$G_{\mathrm{L}}(s)=\lim _{\eta \rightarrow 0} G(z(s)+\eta)=\lim _{\eta \rightarrow 0} \frac{1}{n} \sum_{l} \frac{1}{z(s)-z\left(s_{l}\right)+\eta} \quad \eta=\eta_{x}+\mathrm{i} \eta_{y}$

Expanding around $s_{l}$ we get

$$
\begin{gathered}
\lim _{\eta \rightarrow 0} \frac{1}{z(s)-z\left(s_{l}\right)+\eta}=\lim _{\eta \rightarrow 0} \frac{1}{z^{\prime}\left(s_{l}\right)} \frac{1}{\left[\left(s-s_{l}\right)+\hat{\eta}\right]} \quad \text { where } \hat{\eta}=\frac{\eta}{z^{\prime}\left(s_{l}\right)} \\
=P-\mathrm{i} \pi \frac{\delta\left(s-s_{l}\right)}{z^{\prime}\left(s_{l}\right)} \operatorname{sign}\left(\hat{\eta}_{y}\right)
\end{gathered}
$$

where $P$ denotes the principal part having a continuous variation across the curve. Putting this into equation (24) and using the definition $\rho(s)=\frac{1}{n} \sum \delta\left(s-s_{l}\right)$ we get

$$
\begin{aligned}
G_{\mathrm{L}}(s) & =P-\mathrm{i} \pi \frac{\rho(s)}{z^{\prime}(s)} \operatorname{sign}\left(\hat{\eta}_{y}\right) \\
& =P+\frac{\mathrm{i} \pi \rho(s)}{x^{\prime}(s)+\mathrm{i} y^{\prime}(s)} \operatorname{sign}\left[\eta_{x} y^{\prime}(s)-\eta_{y} x^{\prime}(s)\right] .
\end{aligned}
$$

We note that the above result has a simple geometric meaning: the discontinuity is related to the variation along the normal to the curve. The discontinuity in the Green function across the curve is thus given by

$$
G_{\mathrm{L}}^{+}(s)-G_{\mathrm{L}}^{-}(s)= \pm \frac{2 \pi \mathrm{i} \rho(s)}{z^{\prime}(s)}=2 \pi(\alpha+\mathrm{i} \beta)
$$

where $\alpha$ and $\beta$ define the real and imaginary parts of the jump. Defining the projected densities $\rho_{x}(x)=\frac{1}{n} \sum \delta\left(x-x_{l}\right)=\rho(s) /\left|x^{\prime}(s)\right|$ and $\rho_{y}(y)=\frac{1}{n} \sum \delta\left(y-y_{l}\right)=\rho(s) /\left|y^{\prime}(s)\right|$ we then get

$$
\rho_{x}(x)=\frac{\alpha^{2}+\beta^{2}}{|\beta|} \quad \rho_{y}(y)=\frac{\alpha^{2}+\beta^{2}}{|\alpha|} .
$$

In figure 4 we plot the density of zeros, $\rho(s)$, obtained numerically from equations (19) and (28), with the parameter $s$ chosen as the Euclidean length along the curve. 


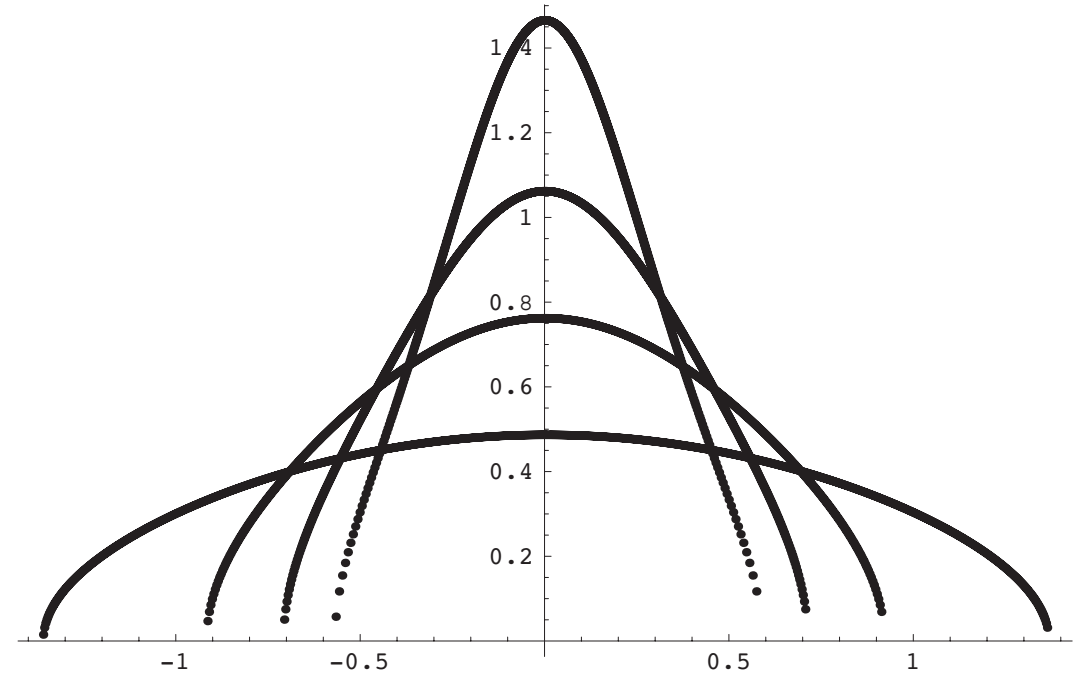

Figure 4. The asymptotic density of zeros $\rho(s)$ as a function of the Euclidean length along the curve for $d=0.2,0.4,0.6$ and 0.8 . The densities are evaluated numerically using the formula in equation (28).

\section{Conclusions}

In summary we have studied a set of coupled nonlinear algebraic equations which are essentially the Bethe ansatz equations for the lowest-energy states of the Heisenberg ferromagnetic chain, at small densities. Both a perturbative and an exact solution of the equations were obtained. In the former case we find that due to some very special properties of the Calogero matrix, we are able to calculate the perturbation series to all orders. The exact solutions of the equations were obtained following a method due to Stieltjes and these are given by the zeros of generalized associated Laguerre polynomials. These zeros are distributed on the complex plane and using Green functions and saddle-point techniques we have obtained the exact asymptotic distribution. It may be noted that there are other examples in physics where one needs to study complex zeros of polynomials as for example in the case of the Yang-Lee zeros. However this is probably one of the few examples where the distribution of zeros in the complex plane has been computed exactly.

\section{Appendix A}

We give an account of Stieltjes electrostatic interpretation of zeros of the orthogonal polynomials [3,4]. The case of the Hermite polynomials is widely known in the physics literature and has been widely applied. This does not seem to be the case for the other orthogonal polynomials.

We first outline the derivation of the electrostatic interpretation for the Hermite polynomial. Consider a set of charges in one dimension interacting by a logarithmic repulsive force and confined within a harmonic potential well. The potential energy of the system is given by

$$
E=\frac{1}{2} \sum_{l=1, n} x_{l}^{2}-\frac{1}{2} \sum_{l \neq m} \ln \left|x_{l}-x_{m}\right|
$$


The minimum of the energy is given by

$$
\partial E / \partial x_{l}=x_{l}-\sum_{m \neq l} \frac{1}{x_{l}-x_{m}}=0 .
$$

Stieltjes' result is that the $n$ zeros of the Hermite polynomial $H_{n}(x)$ satisfy this equation. To show this, consider the $n$ th-degree polynomial

$$
g(x)=\prod_{l=1, n}\left(x-x_{l}\right)
$$

where $x_{l}$ are the $n$ roots of equation (A.2). Taking derivatives with respect to $x$ we get

$$
g^{\prime}(x)=\sum_{l} \prod_{m \neq l}\left(x-x_{m}\right) \quad g^{\prime \prime}(x)=\sum_{k \neq l} \prod_{m \neq k, l}\left(x-x_{m}\right) .
$$

Hence

$$
\frac{g^{\prime \prime}\left(x_{l}\right)}{g^{\prime}\left(x_{l}\right)}=2 \sum_{m \neq l} \frac{1}{x_{l}-x_{m}}=2 x_{l}
$$

since $x_{l}$ satisfy equation (A.2). Thus we have $g^{\prime \prime}\left(x_{l}\right)-2 x_{l} g^{\prime}\left(x_{l}\right)=0$. We further note that $f(x)=g^{\prime \prime}(x)-2 x g^{\prime}(x)$ is a polynomial of degree $n$ with the same roots as $g(x)$ and so they must be identical apart from a constant multiplicative factor. This constant factor is determined by evaluating the coefficient of $x^{n}$ in $f(x)$, which is $-2 n$. We thus obtain $f(x)=-2 n g(x)$ or

$$
g^{\prime \prime}(x)-2 x g^{\prime}(x)+2 n g(x)=0
$$

which is just the equation for the Hermite polynomials. Thus it has been shown that the energy function in equation (A.1) is minimized by a configuration in which the $n$ particles are located at the zeros of the $H_{n}(x)$.

This result can be generalized by replacing $2 x_{l}$ in equation (A.5) by the expression $-q\left(x_{l}\right) / p\left(x_{l}\right)$, where $q(x)$ and $p(x)$ are arbitrary polynomials of degree 1 and 2 respectively. Taking $q(x)=q_{0}+q_{1} x$ and $p(x)=p_{0}+p_{1} x+p_{2} x^{2}$ we repeat the previous arguments to obtain

$$
p(x) g^{\prime \prime}(x)+q(x) g^{\prime}(x)+\left[-n(n-1) p_{2}-n q_{1}\right] g(x)=0 .
$$

Denoting the polynomial solutions of this general equation by $g_{n}(x)$ we have the following result: the zeros of the polynomial $g_{n}(x)$ correspond to the solution of the set of equations

$$
\frac{q\left(x_{l}\right)}{2 p\left(x_{l}\right)}+\sum_{m \neq l} \frac{1}{x_{l}-x_{m}}=0 \text {. }
$$

This corresponds to a minimization of the following $n$-particle potential energy:

$$
E=\frac{1}{2} \sum_{l} \int \frac{q\left(x_{l}\right)}{p\left(x_{l}\right)}+\frac{1}{2} \sum_{l \neq m} \ln \left|x_{l}-x_{m}\right| .
$$

We note that these results are valid for arbitrary complex values of the coefficients $p_{i}$ and $q_{i}$, though a physical interpretation cannot be given in all cases. In fact, as we shall now see, all the cases which give rise to real confining potentials are precisely the ones corresponding to the classical orthogonal polynomials. Physically we expect that for a confining potential there will be real solutions to the minimization problem and this is reflected in the fact that all orthogonal polynomials of the $n$th degree have exactly $n$ real zeros (this of course also follows from the Sturm-Liouville theory). We now list the various cases which result in the standard orthogonal polynomials. 
(i) $p_{2} \neq 0$. In this case, it can be shown that up to linear and scale transformations of the variable $x$, the most general form of the differential equation which leads to a confining potential is the following:

$$
\left(1-x^{2}\right) g^{\prime \prime}(x)+[b-a-(a+b+2) x] g^{\prime}(x)+n(n+a+b+1) g(x)=0 \quad a, b>-1
$$

The resulting potential $V(x)=-(1+a) \ln (1-x)-(1+b) \ln (1+x)$ confines particles within the domain $-1<x<1$. This is the equation for Jacobi polynomials, $P_{n}^{(a, b)}(x)$, of which the Legendre and Chebyshev polynomials are special cases [7].

(ii) $p_{2}=0, p_{1} \neq 0$. In this case the most general form with a bounding potential is

$$
x g^{\prime \prime}(x)+(a+1-x) g^{\prime}(x)+n g(x)=0 \quad a>-1 .
$$

The resulting potential $V(x)=x-(a+1) \ln x$ confines particles to $(0, \infty)$ and corresponds to the associated Laguerre polynomials, $L_{n}^{a}(x)$ [7].

(iii) Finally we have the case $p_{1}=p_{2}=0, p_{0} \neq 0$ and this gives rise to Hermite's equation, which has the harmonic potential $V(x)=x^{2} / 2$.

We note that apart from these special cases the other cases, where $p_{i}, q_{i}$ are allowed to take arbitrary complex values, also may give rise to important physical applications as indeed is so for the example of Bethe roots considered in this paper.

\section{Appendix B}

Here we derive a differential equation satisfied by the Green function. We also show how this can be applied to the case of the other orthogonal polynomials in order to extract the density of zeros in those cases. We start with equation (16) and take a single derivative to get

$$
n \frac{\mathrm{d} G(z)}{\mathrm{d} z}=-\frac{1}{F^{2}}\left(\frac{\mathrm{d} F}{\mathrm{~d} z}\right)^{2}+\frac{1}{F} \frac{\mathrm{d}^{2} F}{\mathrm{~d} z^{2}} .
$$

Now $F(z)=L_{n}^{-n / d-1}(-n \pi z)$ and so satisfies the following Second-order equation (which is equation (A.11) with the substitution $y=-n \pi z$ and $a+1=-n / d$ ):

$$
z \frac{\mathrm{d}^{2} F}{\mathrm{~d} z^{2}}+(-n / d+\pi n z) \frac{\mathrm{d} F}{\mathrm{~d} z}-\pi n^{2} F=0 .
$$

Using this and equation (16) we can eliminate the derivatives on the right-hand side of equation (B.1) to get our equation for the Green function:

$$
\frac{1}{n} \frac{\mathrm{d} G(z)}{\mathrm{d} z}=-G^{2}(z)-\left(\frac{-1}{\mathrm{~d} z}+\pi\right) G(z)+\frac{\pi}{z} .
$$

In the limit $n \rightarrow \infty$, the left-hand side vanishes and the roots of the quadratic on the right-hand side give us the two branches of the Green function. To choose between the branches of course requires a examination of the saddle points as described in section 4 .

This procedure of obtaining the asymptotic Green function is easily generalizable to other polynomial equations and we briefly discuss a few applications to cases where the zeros are real.

(1) Associated Laguerre polynomials with $a+1=p n>0$. The replacement $d=-1 / p$ at once gives us

$$
G(z)=\frac{-\pi z-p \pm \pi \sqrt{\left(z-x_{+}\right)\left(z-x_{-}\right)}}{2 z}
$$


with

$$
x_{ \pm}=(-p-2 \pm 2 \sqrt{p+1}) / \pi .
$$

The roots are located in the region $x_{-}<x<x_{+}$and the density of zeros, given by the discontinuity in the imaginary part of the Green function, is obtained as

$$
\rho(x)=\frac{1}{2 x} \sqrt{\left(x_{+}-x\right)\left(x-x_{-}\right)} .
$$

We can also consider the case where the coefficient $a$ does not scale with $n$ but is a finite constant. The Green function and density of zeros are found to be independent of $a$ :

$$
\begin{aligned}
& G(z)=\frac{-\pi}{2}\left[1-\sqrt{1+\frac{4}{\pi z}}\right] \\
& \rho(x)=\frac{1}{2} \sqrt{\frac{4}{\pi|x|}-1 \quad-4 / \pi<x<0 .}
\end{aligned}
$$

(2) Hermite polynomials. In this case we use scaled variables such that the zeros $z_{l}$ satisfy $H_{n}\left(\sqrt{n} z_{l}\right)=0$. Proceeding as before we get the following equation for the Green function:

$$
\frac{1}{n} \frac{\mathrm{d} G(z)}{\mathrm{d} z}=-G^{2}(z)+2 z G(z)-2 .
$$

The asymptotic Green function and the density of zeros follow immediately:

$$
\begin{aligned}
& G(z)=z-\sqrt{z^{2}-2} \\
& \rho(x)=\frac{1}{\pi} \sqrt{2-x^{2}} .
\end{aligned}
$$

(3) Jacobi polynomials. If $z_{l}$ satisfy $P_{n}^{(a, b)}\left(z_{l}\right)=0$, then the corresponding equation for the Green function is

$$
\frac{1}{n} \frac{\mathrm{d} G(z)}{\mathrm{d} z}=-G^{2}(z)-\frac{b-a-(a+b+2) z}{n\left(1-z^{2}\right)} G(z)-\frac{n+a+b+1}{n\left(1-z^{2}\right)} .
$$

We thus get the following asymptotic Green function and density of states:

$$
\begin{aligned}
& G(z)=\frac{1}{\sqrt{z^{2}-1}} \\
& \rho(x)=\frac{1}{\pi \sqrt{1-x^{2}}} \quad-1<x<1 .
\end{aligned}
$$

The results in equations (B.5)-(B.7) are identical to those given in [8], where they have been obtained by different methods.

\section{References}

[1] Dhar A and Shastry B S 2000 Phys. Rev. Lett. 852813

[2] Mehta M L 1991 Random Matrices (London: Academic)

[3] Stieltjes T J 1914 Sur Quelques Theoremes d'Algebre, Oeuvres Completes vol 1 (Groningen: Noordhoff) p 440

[4] Szego G 1975 Orthogonal Polynomials 4th edn (Providence, RI: American Mathematical Society)

[5] Calogero F 1981 J. Math. Phys. 22919

[6] Muttalib K A 1990 Phys. Rev. Lett. 65745

[7] Gradshteyn I S and Ryzhik I M 1994 Table of Integrals, Series and Products (London: Academic)

[8] Lang W 1997 Preprint arXiv math.CA/9711217

[9] Chen L C and Ismail M 1991 SIAM J. Math. Anal. 221442

[10] Andrews G E and Askey R A 1985 Lecture Notes in Maths, 1171 (Berlin: Springer) pp 36-62

[11] Dette H and Studden W J 1994 Preprint arXiv math.CA/9406224 